\title{
Cracking Behavior in Additively Manufactured Pure Tungsten
}

\author{
Dian-Zheng Wang ${ }^{1} \cdot K_{\text {Kai-Lun } \mathrm{Li}^{1} \cdot \text { Chen-Fan } \mathrm{Yu}^{1} \cdot \mathrm{Jing} \mathrm{Ma}^{1} \cdot \text { Wei Liu }}{ }^{1} \cdot \mathrm{Zhi}^{-J i a n}$ Shen $^{1,2}$
}

Received: 22 February 2018/Revised: 27 March 2018/ Published online: 19 May 2018

(C) The Chinese Society for Metals and Springer-Verlag GmbH Germany, part of Springer Nature 2018

\begin{abstract}
In this study, near fully dense (96.5\%) pure tungsten bulks were additively manufactured and the cracking behavior was investigated. A crack network with a spacing of $\sim 100 \mu \mathrm{m}$ was observed in the fabricated bulks. It was observed that the laser scanning strategy, which could tailor the microstructure, affected the crack distribution pattern in fabricated tungsten. The calculated surface temperature difference $(7300 \mathrm{~K})$ was much higher than the cracking criterion $(800 \mathrm{~K})$ of tungsten, indicating that cracking is almost inevitable in laser additive manufacturing of tungsten. It could be concluded that crack network formed because the cracks emerged in every laser molten track and then interconnected in the layer-by-layer building process.
\end{abstract}

Keywords Tungsten $\cdot$ Selective laser melting $\cdot$ Cracking $\cdot$ Microstructure

\section{Introduction}

Possessing high melting point $(3693 \mathrm{~K})$, high thermal conductivity, and strong mechanical properties, tungsten has been widely used in military and electrical industries [1]. In the international thermonuclear experimental reactor (ITER), tungsten is considered as the most promising candidate for plasma-facing materials owing to its high resistance to irradiation and low erosion yield [2]. Due to the high brittleness, tungsten parts are difficult to be mechanically processed and are conventionally fabricated via powder metallurgy method, which encounters some difficulties in the production of a complex structure. Therefore, additive manufacturing (AM) of tungsten, which can produce metal parts with complex inner and outer structures, has attracted the attention of many

Available online at http://link.springer.com/journal/40195

Wei Liu

liuw_tsinghua@126.com

$\triangle$ Zhi-Jian Shen

shenzhijian@tsinghua.edu.cn

1 State Key Laboratory of New Ceramic and Fine Processing, School of Materials Science and Engineering, Tsinghua University, Beijing 100084, China

2 Department of Materials and Environmental Chemistry, Arrhenius Laboratory, Stockholm University, 10691 Stockholm, Sweden researchers. Zhang et al. [3] and Zhou et al. [4] tried to additively manufacture pure tungsten parts and found that the densification is difficult due to the balling phenomenon. Deprez et al. [5] fabricated a tungsten collimator component for computed tomography equipment; only a density of $89.2 \%$ was achieved. As an AM technology, selective laser melting (SLM) is popular owing to its ability to produce high-precision metal parts and has been applied to many materials, including stainless steel $[6,7]$, titanium alloy [8, 9], and even superalloy [10]. Wang et al. [11] successfully applied SLM to fabricate near fully dense pure tungsten parts (96.0\%), in which many cracks were observed. In fact, the cracking problem has been widely reported in SLM process. Due to the thermal stress in SLM, even ductile material like 316L can nucleate cracks [12]. Qian et al. [13] also reported the cracking in SLM-derived CoCrMo alloy. Li et al. [14] reported the cracking in SLM of Al-based metallic glass and found that laser power may affect the crack density. Some Ni-based superalloy is sensitive to cracking in SLM and has been widely researched $[15,16]$. However, cracking in additively manufactured tungsten has rarely been reported, though it is one of the main challenges.

SLM can be treated similarly to laser or electron irradiation process, in which the cracking behavior of tungsten has been widely investigated. Huber et al. [17] investigated the thermal shock response of tungsten to multi-laser pulse and observed that the crack could penetrate the melting layer, forming a crack net. Similar cracking behavior was 
also reported by Farid et al. [18], who investigated the cracking behavior under long laser pulse load. Wirtz et al. $[19,20]$ tested the thermal shock impact of an electron beam on tungsten and further proved that the microstructure would affect the crack distribution and density. In the SLM process, the laser parameters, especially the scanning strategy, have a considerable influence on the final microstructure and thus affect the cracking behavior. Carter et al. [21] conducted the SLM of nickel superalloy CM247LC and observed that the final bulk could be divided into heavy and light crack regions, which was determined using scanning strategies. Wang et al. [22] reported the cracking in SLM-derived molybdenum and observed that the scanning rotation between layers could intertwine the grain boundary (GB) structure and contribute to crack control.

It is well known that tungsten is susceptible to cracking, which can be ascribed to its high ductile-brittle transition temperature $\left(400{ }^{\circ} \mathrm{C}\right)$ [23]. Under laser irradiation, the driving force of cracking is the inner stress induced by temperature mismatch, whereas the cracking resistance of tungsten mainly depends on its intrinsic ductility as simulated by Qi and Chrzan [24] and Romnar et al. [25]. Though the intrinsic ductility of pure tungsten is hard to be improved, the stress can be tailored because the inhomogeneous temperature field leading to inner stress is determined by the laser parameters and substrate preheating $[26,27]$. In the laser irradiation process of tungsten, it is commonly observed that the power density has a decisive effect on cracking [14, 28], which means that cracking can appear only when the power density is higher than the threshold value. In the SLM process of tungsten, however, the laser power density should be sufficiently high to reach densification; therefore, evaluating the cracking tendency based on laser parameters may provide an explanation for the cracking behavior in the SLM of tungsten.

In this study, we additively manufactured pure tungsten parts via SLM and investigated the resulted cracking behavior. Crack distribution and its relation with the microstructure were revealed using scanning electron microscopy (SEM) and electron backscatter diffraction (EBSD). The cracking criterion of tungsten under singlelaser irradiation was calculated and compared with the processing conditions of SLM to explain the formation of crack network.

\section{Experimental}

Spherical powders (purity $>99.9 \%$, median diameter $=28 \mu \mathrm{m}$ ) were used in this study as shown in Fig. 1 . The powders were spheroidized using a plasma spheroidization machine (Teksphero-40, Tekna Co. Ltd, Sherbrooke, Canada) as described in Ref. [29].

The SLM experiments were conducted using a Renishaw AM400 machine (Renishaw, New Mill, UK) at a maximum power of $400 \mathrm{~W}$ with a Nd/YAG fiber laser having a spot size of $75 \mu \mathrm{m}$. Three types of scanning strategies were applied in this study: (1) the scanning direction was fixed in all the layers (Fig. 2a); (2) the scanning direction was rotated by $67^{\circ}$ between layers (Fig. 2b); (3) besides the scanning rotation, the layer was melted twice in every layer (Fig. 2c). The laser was scanned in a "zigzag" manner under pulse mode (Fig. 2d), which means that the laser was exposed to one point for a short period and thereafter moved to the next point. The optimized parameters were as follows: laser power of $400 \mathrm{~W}$, point distance of $75 \mu \mathrm{m}$, exposure time of $200 \mu \mathrm{s}$, hatch distance of $100 \mu \mathrm{m}$, and layer thickness of $30 \mu \mathrm{m}$. To limit the oxidation of tungsten, the fabrication process was conducted in a chamber filled with argon gas to maintain the oxygen content below $100 \mathrm{ppm}$. A steel substrate was used in the SLM experiments, and no preheating was performed. Under the three scanning strategies (1)-(3), cubic tungsten bulks of $10 \mathrm{~mm} \times 10 \mathrm{~mm} \times 5$ $\mathrm{mm}$ were fabricated (Fig. 2e) and their final densities reached 92.5, 96.0, and 96.5\%, respectively.

The specimens were microscopically characterized using a TESCAN MIRA 3LMH SEM (TESCAN, Brno, Czech Republic) equipped with an EBSD detector (Oxford Instruments, Oxford, UK) and an HKL Nordlys orientation imaging microscope system. The EBSD data were processed using HKL Channel 5 software packages.

\section{Results}

\subsection{Crack Network in SLM-Derived Tungsten}

First of all, a single tungsten molten track was fabricated on a rolled tungsten substrate as shown in Fig. 3. A continuous track with a regular surface ripple, which originated from the oscillation of the melting pool, was obtained after laser scanning. The surface ripples also indicated the scanning direction (SD, the black arrows). Because the laser worked in the pulse mode, the surface ripples were fluctuant at some positions (red arrows). It can be observed that obvious cracks emerged after the laser scanning. On the top face (Fig. 3a), a major crack grew along the scanning direction, whereas some micro-crack nucleated from the major crack (local amplified image). On the side face (Fig. 3b), the crack grew deep into the substrate and reached $200 \mu \mathrm{m}$, which is much larger than the depth of the melting pool. The cracking in single-track scanning of SLM was also reported in SLM of Al-based metallic glass 


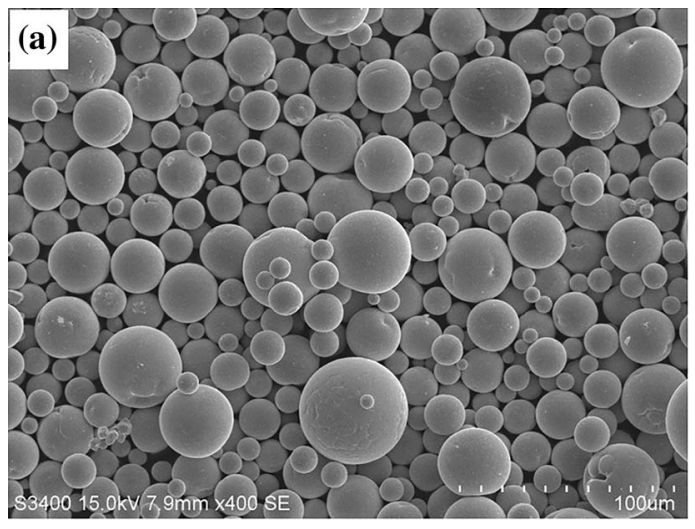

Fig. 1 a SEM image and $\mathbf{b}$ size distribution of raw tungsten powders
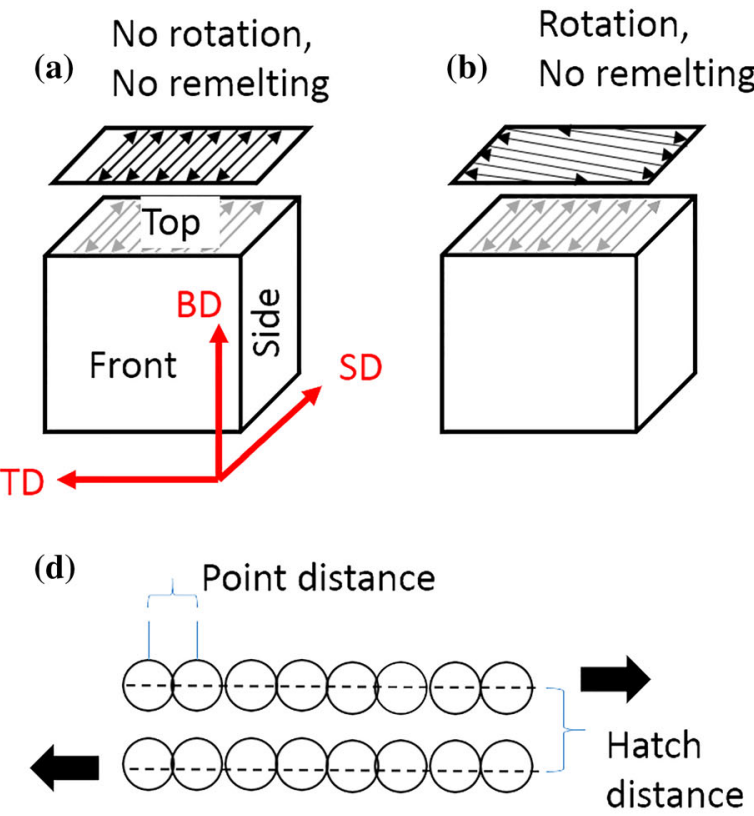

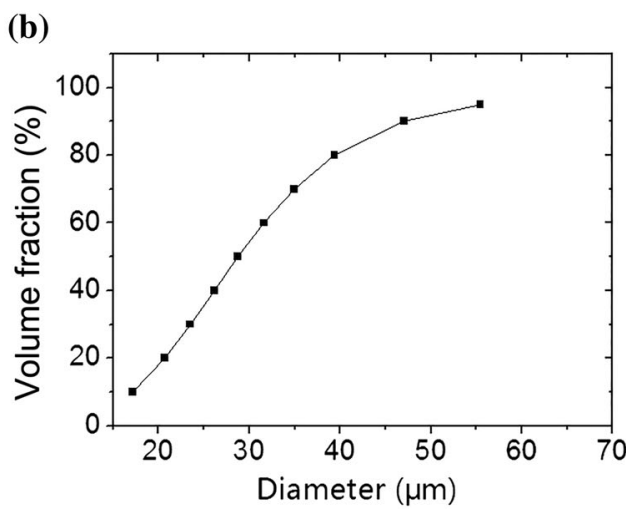

(b)
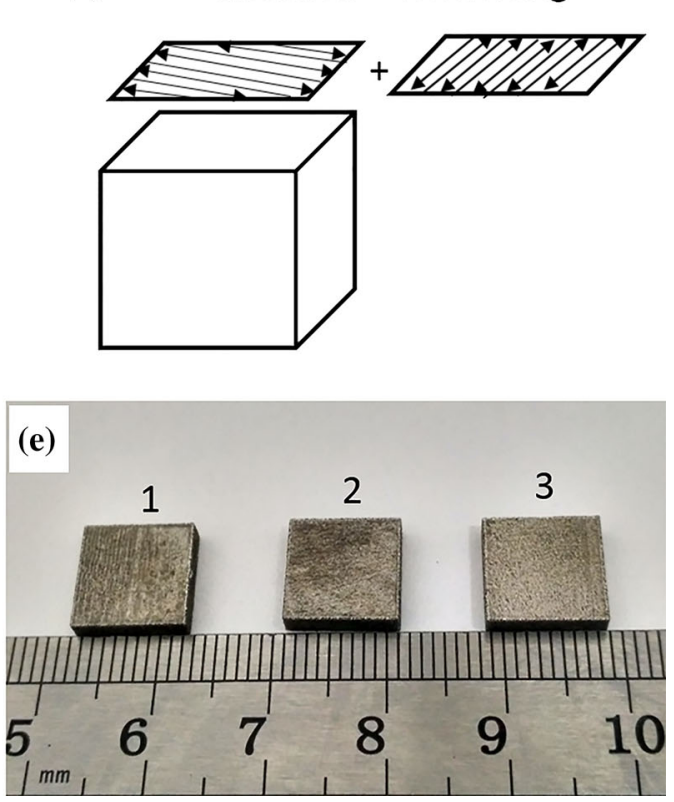

Fig. 2 Schematic illustration of scanning strategies of a no scanning rotation, no remelting, $\mathbf{b}$ scanning rotation, no remelting, $\mathbf{c}$ scanning rotation and remelting, $\mathbf{d}$ illustration of pulse laser mode, e picture of pure tungsten bulks manufactured under scanning strategies (1)-(3) in order from left to right (BD: building direction; SD: laser scanning direction; TD: transverse direction)

by Li et al. [14]. The cracking in single molten track also matches well with the thermal shock tests of tungsten $[19,20]$.

As illustrated in Fig. 4, the SLM-derived tungsten bulk exhibits smooth surface morphology with continuous molten tracks of width $100 \mu \mathrm{m}$ (white dash lines), indicating high density $(96.0 \%)$. The laser SD can also be deduced according to the surface ripples (black arrows). However, an apparent crack network can be observed on the surface as indicated. The cracks can be divided into two types: longitudinal crack (pair of red arrows), located at the center of molten tracks and parallel to SD, transverse crack (blue lines), which intersects in a slant manner with SD and forms a "V" shape. Quite similar crack network was observed in SLM-derived pure molybdenum [22].

On the side face, it can be observed that the cracks grow deep into several layers (thickness $30 \mu \mathrm{m}$ in this study), indicating that they can affect several layers. Owing to insufficient overlapping of the molten track [30], some residual pores can be observed as shown by red arrows in Fig. 5. As mentioned in Ref. [6], the pore defects may nucleate cracks as indicated by the red circles in Fig. 5. A crack network can be observed with a spacing of $\sim 100 \mu \mathrm{m}$, which is equal to hatch distance, in tungsten fabricated via laser AM. 


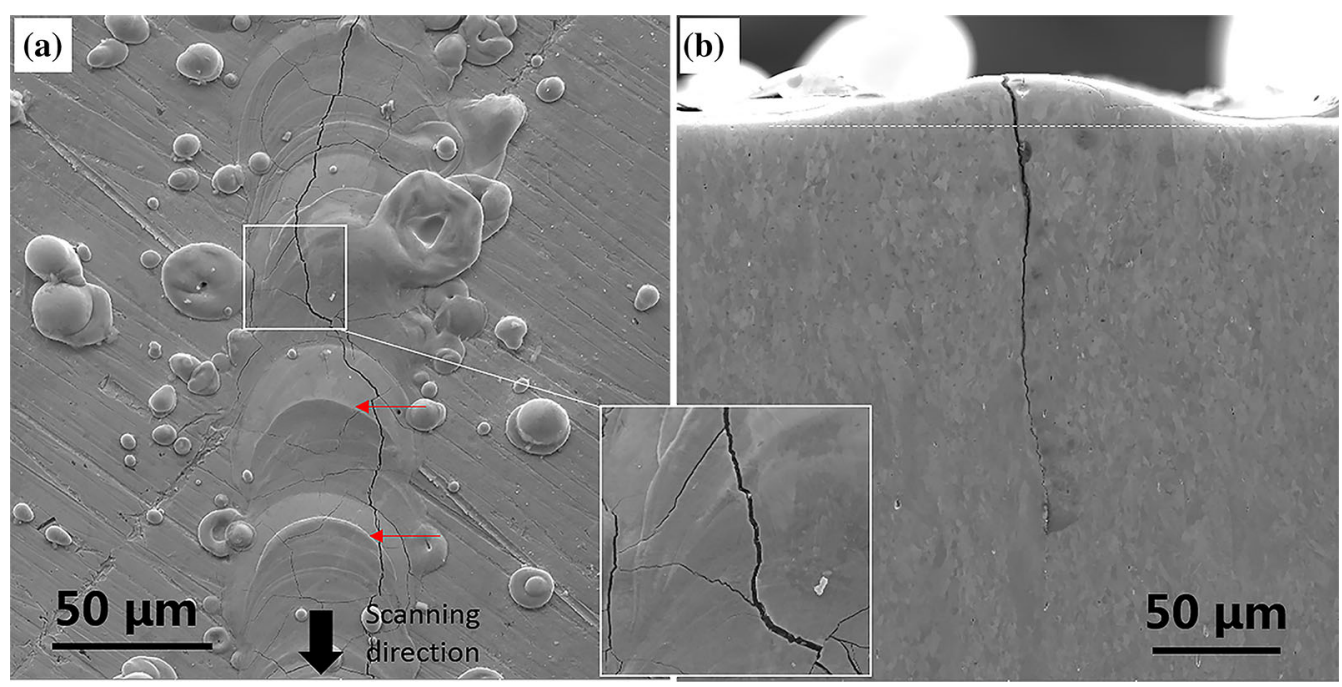

Fig. 3 SEM images showing cracking in a single-laser molten track of tungsten: $\mathbf{a}$ top face, $\mathbf{b}$ front face

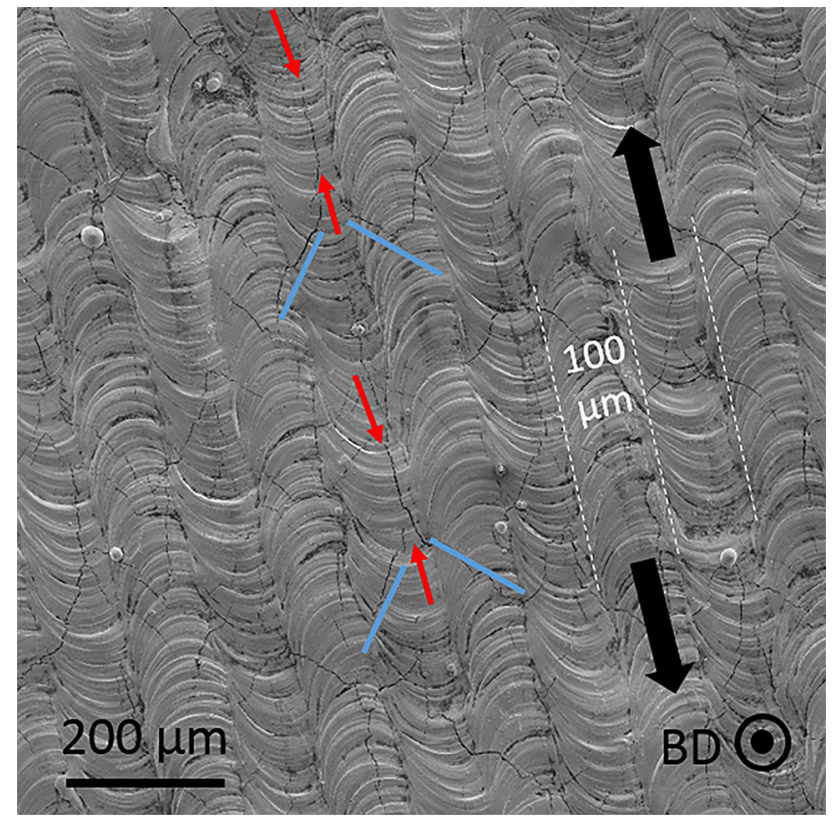

Fig. 4 Crack distribution in top face of built pure tungsten (the sample was fabricated under laser scanning rotation of $67^{\circ}$ without remelting)

\subsection{Grain Boundary Growth Behavior of Cracks}

In order to investigate the relation between crack growth and the GB structure, EBSD was performed and the result is shown in Fig. 6. It is well known that the cracks in tungsten tend to grow at the GB due to its weakness [23]. Further, it can be observed that the cracks grow selectively. First, cracks tend to grow along high-angle GBs. For example, as indicated by the two slender grains A and B, the cracks tend to grow at a GB with misorientation higher than $40^{\circ}$ rather than at a GB with misorientation of

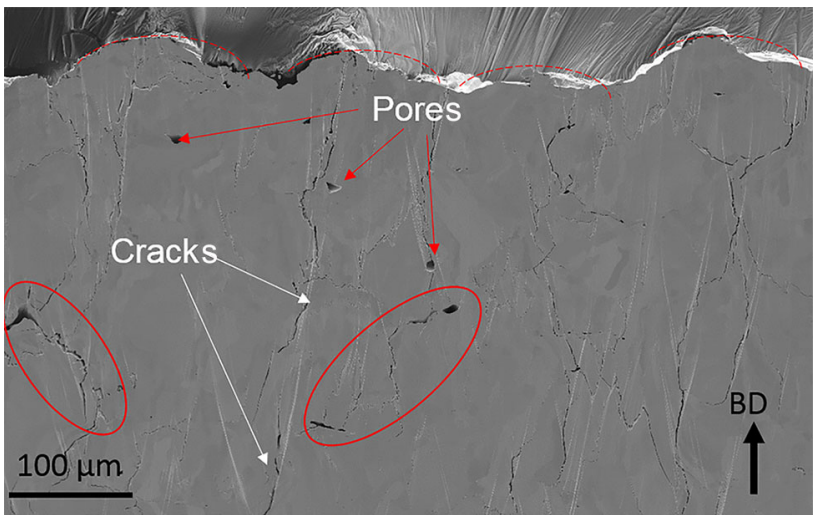

Fig. 5 Crack distribution in side face of built pure tungsten (the sample was fabricated under laser scanning rotation of $67^{\circ}$ without remelting)

approximately $20^{\circ}$. GB D has very low misorientation of $7.5^{\circ}$; thus, the crack stops at this GB. The fact that cracking resistance increased with GB misorientation is also reported in Ref. [16]. Second, the cracks can be suppressed by the coincidence site lattice (CSL) GB. For example, GB C possesses $55 \mathrm{CSL}$ structure; hence, the grain does not propagate along this GB despite its high misorientation $\left(42^{\circ}\right)$. The above two crack growth preferences agree with the tungsten GB strength tests provided by Mikhailovskij et al. [31]. The Zone $\mathrm{E}$ shows the distortion of orientation at crack tip, caused by the localized plastic deformation during crack growth. The deformation at crack tip is the main cracking growth resistance [32].

\subsection{Effect of Scanning Strategy on Crack Growth}

Figure 7 illustrates the cracking in SLM-derived tungsten without scanning rotation or remelting. On the top face 


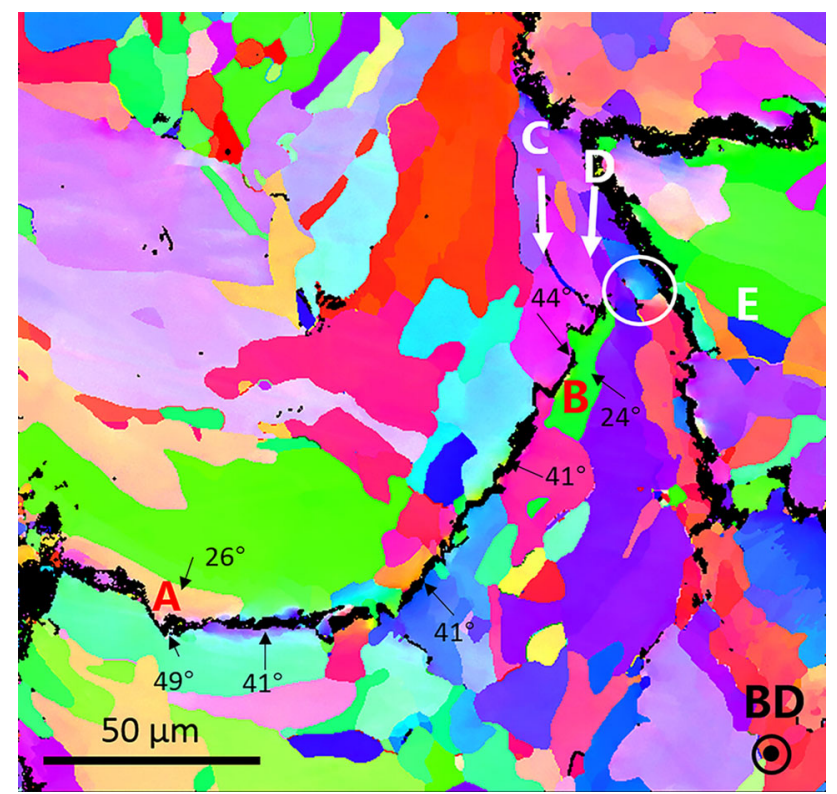

Fig. 6 EBSD map showing crack growth at GB (A and B are two grains; $\mathrm{C}$ and $\mathrm{D}$ are two GBs; $\mathrm{E}$ presents the local deformation area)

(Fig. 7a), the longitudinal (red arrows) and transverse cracks (blue lines) are evident. From the inverse pole figure (IPF) mapping (Fig. 7b), it can be observed that the grains are arranged with a strong regulation and exhibit a "ladder" shape, namely the slender grains are stacked together and form many parallel grain rows. The scanning direction can be deduced according to the characteristic grain morphologies [33] as shown by the black arrows. Inside the laser melting pool (black arc in Fig. 7b), the grains grow along the maximum temperature gradient, which is normal to the black arc line, forming slender grains. The slender grains come into contact at the center of melting pool and form the central GB along the molten track. With the movement of the laser spot, the central GB elongates and forms the longitudinal GB (white line in Fig. 7b). The longitudinal GB and relevant crack at the center of molten track are similar to the laser welding process as observed in Ref. [34]. Notably, the molten track observed in Fig. 4 cannot be traced in the IPF map owing to the epitaxial growth of the grains. In Fig. 7b, the black dash lines indicate the supposed boundaries of the molten tracks.

On the front face (Fig. 7c), the cracks grow along the $\mathrm{BD}$ and the crack can be elongated over $1 \mathrm{~mm}$. From the IPF mapping (Fig. 7d), it can be observed that the columnar grains induced by directional solidification grow along the BD. The columnar grains are almost parallel to each other, forming the vertical GBs along the BD. In fact, the columnar grains are formed via two adjacent laser scans in one layer. The dash white line pairs indicate the
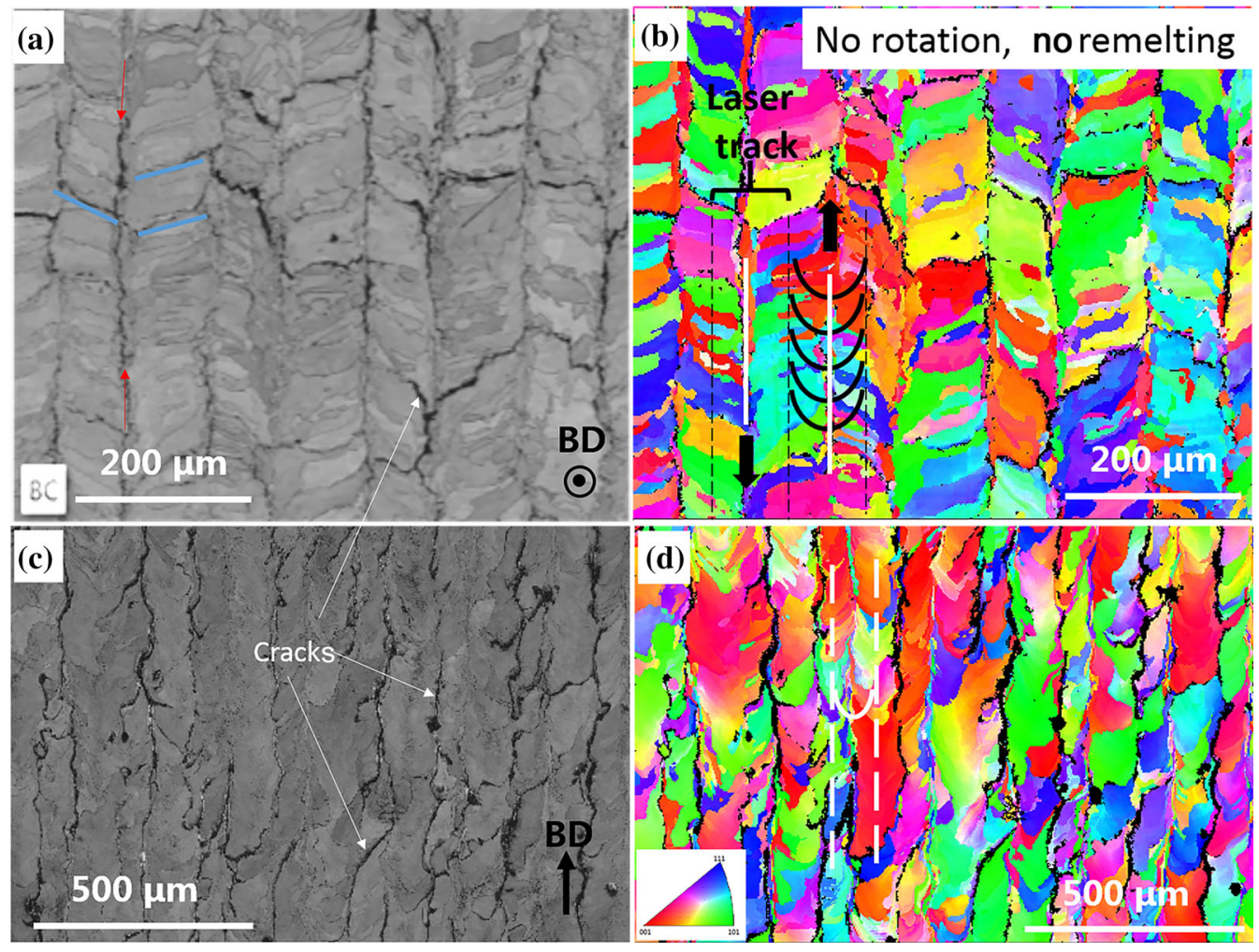

Fig. 7 Cracking in tungsten fabricated with no scanning rotation and no remelting: a SEM image of top face; b EBSD mapping of a; c SEM image of side face; $\mathbf{d}$ EBSD mapping of $\mathbf{c}$ 
supposed position of the laser track, whereas the semicircle indicates the boundary of the melting pool. Inside the laser melting pool, the grains grow in a direction normal to the semicircle. Eventually, the grains encounter each other at the center of the melting pool, forming the vertical GB, which provides the growth path for cracks. Combined with the morphology of the top face (Fig. 7b), it can be predicted that the columnar grains are plate-like structures.

When the SD was rotated by $67^{\circ}$ between layers, the grains on the top face became disordered as shown in Fig. 8a. Furthermore, the angle of $67^{\circ}$ was chosen to avoid the repetition of SD as much as possible. Owing to the scanning rotation, the ladder-shaped grain arrangement on the top face was partially disturbed (Fig. 8a). Similar results were also observed in the SLM of molybdenum [22], CoCrMo alloy [33]. The trace of molten tracks can be observed at some positions as shown by the white dash lines under this condition. On the side face (Fig. 8b, c), the boundary structure of the columnar grains became intertwined due to the scanning rotation. The width of the columnar grains was not uniform throughout the side face.

In order to investigate the effect of remelting, the deposited powder layer was melted accompanying scanning rotation and the resulting bulks were microstructurally characterized as shown in Fig. 9. Notably, the direction of secondary scanning was vertical to that of the primary scanning. On the top face (Fig. 9a), it could be observed that the characteristic slender grains disappeared completely, demonstrating that the remelting could apparently contribute to the reconstruction of grain morphology. The straight longitudinal cracks disappeared under this condition. No apparent grain refinement was observed because the fast cooling condition is similar to the primary solidification process. On the side face (Fig. 9b, c), the crack still grew deep into several layers. The epitaxial growth tendency could still be observed on the side face, indicating the fast cooling solidification condition.

\section{Discussion}

\subsection{High Cracking Tendency in SLM-Derived Tungsten}

In order to understand the high cracking tendency of tungsten, it is essential to identify the cracking criterion. Due to the uneven heating and cooling process, complex stress field will appear in the SLM process [14, 35]. Moreover, the remelting and reheating would lead to the evolution of stress field. The stress in SLM is theoretically calculated in Ref. [36] and simulated in Ref. [37].

According to the single-track experiment (Fig. 3), it can be deduced that cracks occur in the individual laser
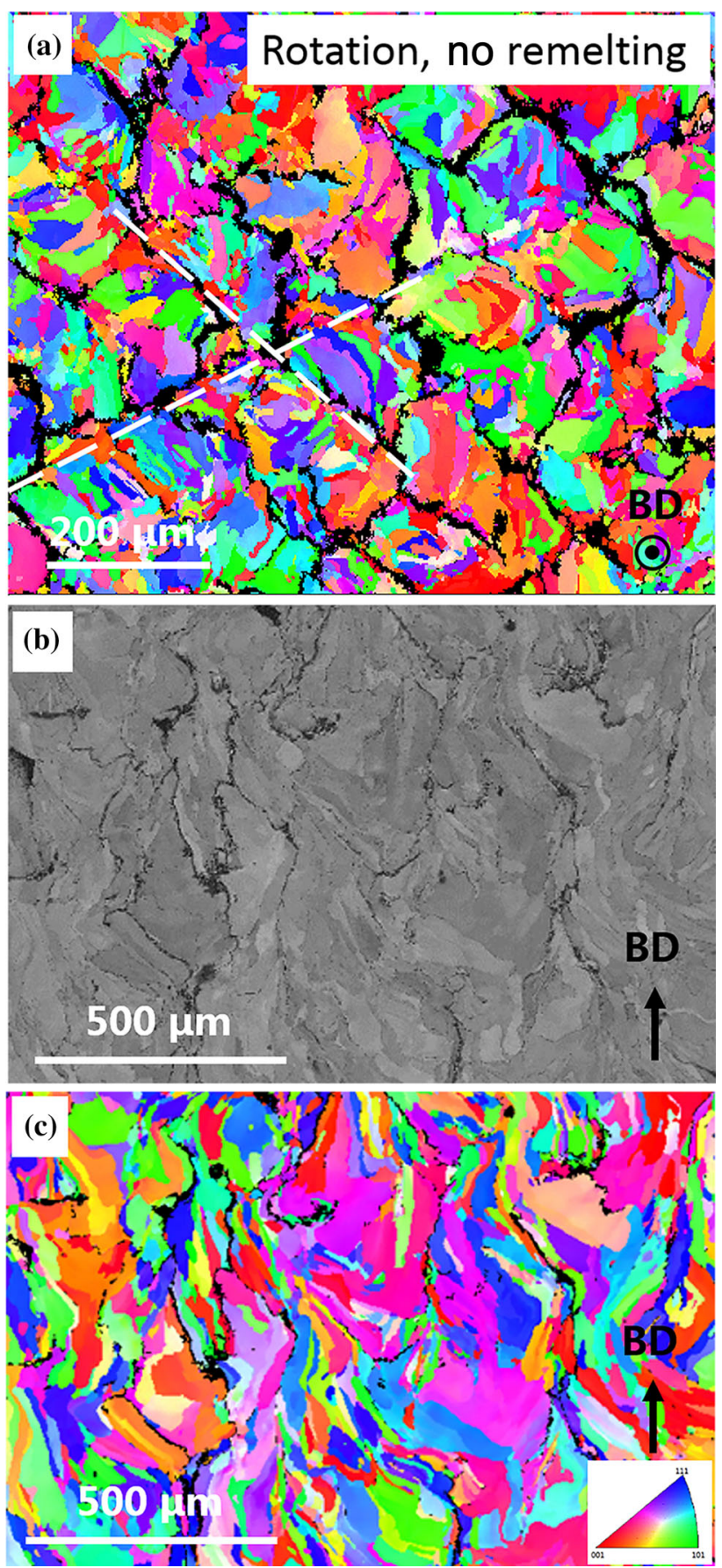

Fig. 8 Cracking in tungsten fabricated under scanning rotation of $67^{\circ}$ without remelting: a EBSD mapping of top face; $\mathbf{b}$ SEM image of side face; $\mathbf{c}$ EBSD mapping of $\mathbf{b}$

scanning process. Considering the laser worked in pulse mode in this study, the SLM process can be simplified as numerous individual laser heat radiation processes. Then, the cracking model can be simplified as the competence between thermal stress and material toughness during oneheat radiation process. The magnitude of thermal stress $\left(\sigma_{\text {therm }}\right)$ can be expressed as [38]: 

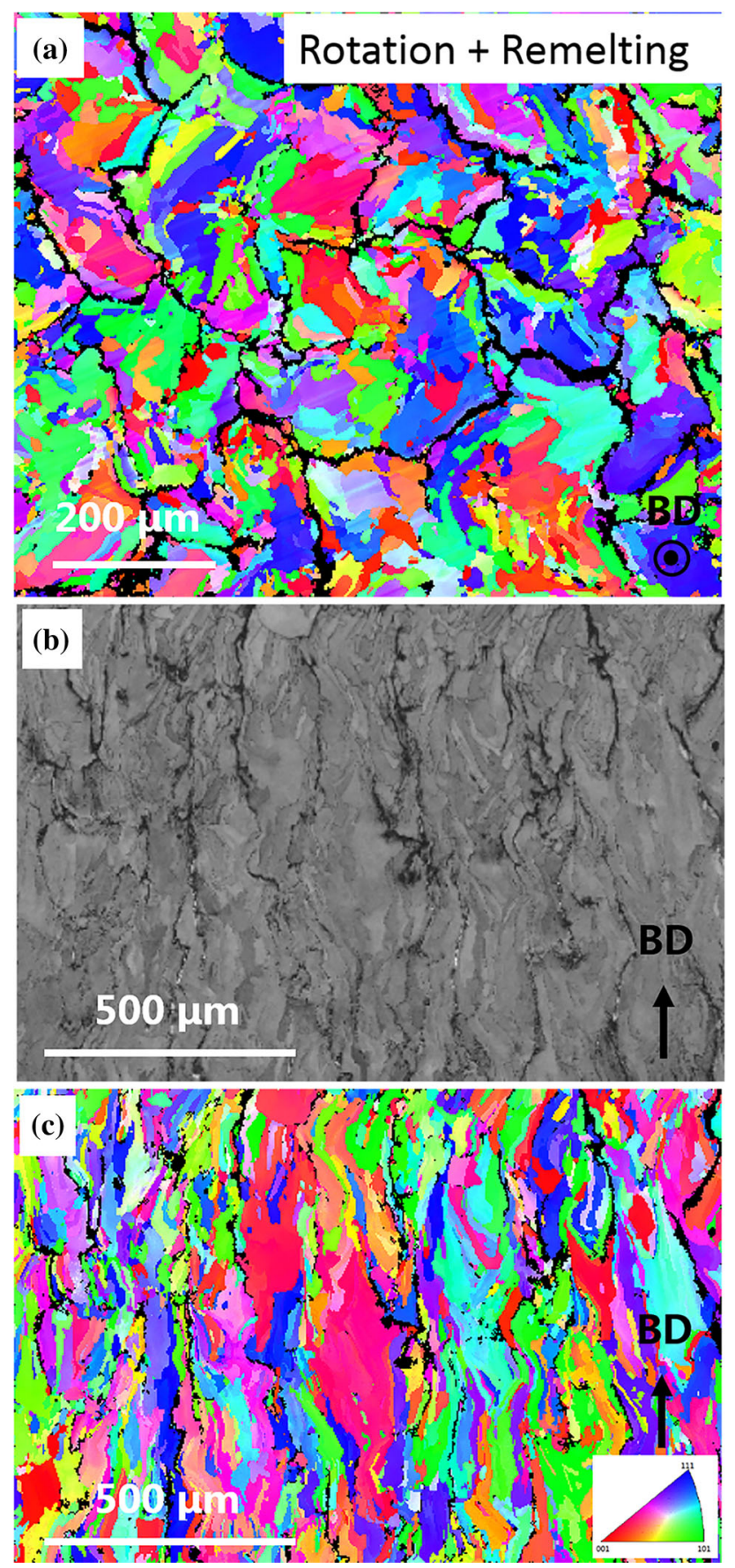

Fig. 9 Cracking in tungsten fabricated under scanning rotation of $67^{\circ}$ with remelting (the remelting SD is vertical to primary SD): a EBSD mapping of top face; $\mathbf{b}$ SEM image of side face; $\mathbf{c}$ EBSD mapping of b

$\sigma_{\text {therm }}=\frac{E \alpha}{1-v} \Delta T$

where $\Delta T$ is the temperature difference, $\alpha$ is the liner expansion coefficient, $v$ is the Poisson's ratio, $E$ is the modulus. If the inner thermal stress exceeds the ultimate tensile strength $\left(\sigma_{\mathrm{UTS}}\right)$, the cracks are initiated: $\sigma_{\text {therm }}>\sigma_{\mathrm{UTS}}$.

By substituting (1) into (2), the cracking criterion can be expressed as:

$\sigma_{\mathrm{UTS}} \frac{1-v}{E \alpha}>\Delta T$.

Notably, the parameters in the left side of Eq. (3) are all material properties, representing the cracking formation resistance of a material. The parameter in the right side of Eq. (3) represents the loaded cracking driving force and depends on the laser parameters, atmospheric temperature, etc. For tungsten, even by adopting the highest reported tensile strength of $2 \mathrm{GPa}$ [1], the maximum endurable temperature difference is calculated as only $800 \mathrm{~K}$ according to Eq. (3). Under laser radiation of SLM, the surface temperature difference can be estimated by the equation as follows:

$\Delta T=2 \sqrt{\frac{\tau}{\pi C_{\mathrm{p}} D}} W$,

where $W$ is the laser power density, $\tau$ is the pulse duration, $C_{\mathrm{p}}$ is the heat capability, and $D$ is the laser diameter. Referring to the laser parameters of tungsten SLM, the temperature difference is calculated as $7300 \mathrm{~K}$, which is much higher than the critical temperature gradient. Thus, the cracking in tungsten fabricated via SLM is almost inevitable. However, considering that the melting point of tungsten is only $3693 \mathrm{~K}$, the real temperature difference in tungsten fabricated via SLM cannot reach $7300 \mathrm{~K}$. Moreover, the preheating has decisive effects for cracking of tungsten. The high cracking tendency can be experimentally proved by the continuity and depth of a longitudinal crack in a single molten track (Fig. 3).

\subsection{Formation of Crack Network}

The formation of longitudinal and transverse crack patterns (Fig. 4) can be ascribed to the characteristic microstructure after the SLM process. As illustrated in Fig. 10, in the melting pool, liquid becomes solidified along the temperature gradient in a directional manner, forming the slender grains (also shown in Fig. 7b). The slender grains meet together in the center of the molten track, forming the longitudinal GBs. The longitudinal and transverse GBs (blue lines in Fig. 10) eventually act as the growth paths of cracks (black lines). When the SD was rotated between layers, the grains were partially disordered as shown in Fig. 8a; therefore, the longitudinal cracks became shorter, whereas the transverse cracks retained the same pattern. The remelting disturbs the grain arrangement on the top face. Consequently, the surface crack is demonstrated to be irregular (Fig. 9a). 


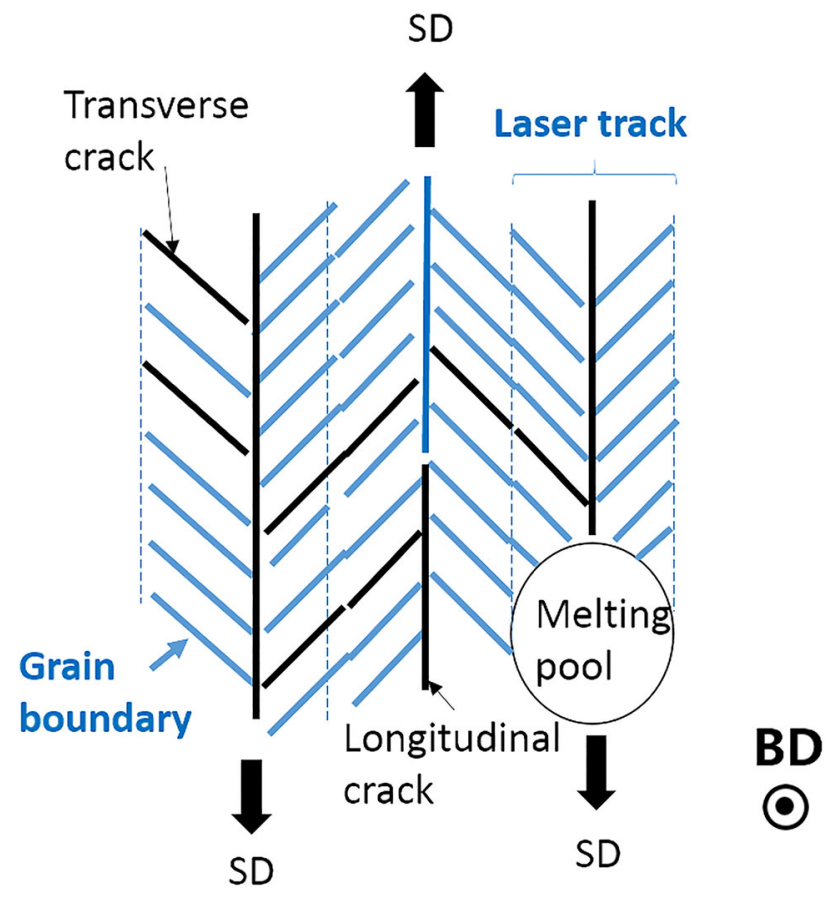

Fig. 10 Schematic illustration of crack pattern and microstructure after SLM

On the side face, directional columnar grains were observed when the SD was fixed (Fig. 7b, c) under the directional solidification mechanism. The vertical GB structure led to vertical cracks, with spacing of $100 \mu \mathrm{m}$ (hatch space) between them. When the SD was rotated between layers, the melting pool was more likely to cover the GBs of the previous layer; therefore, the epitaxial growth was broken and columnar grains became intertwined. The intertwining of the GB structure can contribute to the control of the crack because cracks would encounter more growth resistance (Fig. 8b, c). When the parts are melted twice, the columnar grains can still be observed. However, remelting cannot suppress the cracks (Fig. 9b, c) because the cracks can penetrate the deposition layers.

It can be concluded that longitudinal and transverse cracks would emerge in every molten track. Hirai et al. [28] observed that even a single heat pulse can induce a crack network in tungsten, which is consistent with the results of this study. In the scanning process, cracking occurs track by track, which explains the crack distance of $\sim 100 \mu \mathrm{m}$ observed in Fig. 5. Repeated laser exposure will also cause the fatigue growth of cracks. In the additive deposition process of layers, the cracks would penetrate the new layer and connect with each other, eventually forming a crack network

In the future, measures should be undertaken from the respects of both cracking driving force and resistance to control cracking. Considering the stress is caused by thermal mismatch, substrate preheating has been proven to be an efficient method to reduce the residual stress and control cracks in SLM as mentioned in Ref. [26, 27, 39]. The cracking resistance is mainly determined by its plastic deformation at the crack tip, which can be improved by an alloying approach as mentioned in Ref. [24, 25, 32].

\section{Conclusions}

(1) A crack network with a distance of $\sim 100 \mu \mathrm{m}$ was observed in the SLM-derived tungsten. The cracks grew mainly along the GB. On the top face, there were two kinds of cracks: longitudinal and transverse cracks. The longitudinal cracks grew at the centerline of the molten tracks, whereas the transverse cracks intersected in a slant manner with the molten tracks. On the side face, the cracks grew deep into several layers.

(2) The scanning rotation could contribute to crack control, particularly for longitudinal cracks, because it could intertwine the GB structure and increase the crack growth resistance. The remelting could disturb the grain growth and change the crack pattern, but could not suppress the cracking.

(3) The calculated surface temperature difference $(7300 \mathrm{~K})$ in the SLM process was much higher than the critical cracking value of tungsten ( $800 \mathrm{~K}$ ), indicating that cracking is almost inevitable. Cracks emerged in every laser scanning track and connected with each other, forming a crack network.

Acknowledgements This work was supported financially by the National Magnetic Confinement Fusion Science Program of China (No. 2014GB117000) and the National Natural Science Foundation of China (No. U1605243).

\section{References}

[1] L. Huang, L. Jiang, T.D. Topping, C. Dai, X. Wang, R. Carpenter, C. Haines, J.M. Schoenung, Acta Mater. 122, 19 (2017)

[2] R.A. Pitts, S. Carpentier, F. Escourbiac, T. Hirai, V. Komarov, S. Lisgo, A.S. Kukushkin, A. Loarte, M. Merola, A.S. Naik, R. Mitteau, M. Sugihara, B. Bazylev, P.C. Stangeby, J. Nucl. Mater. 438, S48 (2013)

[3] D.Q. Zhang, Q.Z. Cai, J.H. Liu, Mater. Manuf. Process. 27, 1267 (2012)

[4] X. Zhou, X.H. Liu, D.D. Zhang, Z.J. Shen, W. Liu, J. Mater. Process. Technol. 222, 33 (2015)

[5] K. Deprez, S. Vandenberghe, K. Van Audenhaege, J. Van Vaerenbergh, R. Van Holen, Med. Phys. 40, 1 (2013)

[6] Y. Zhong, L.F. Liu, S. Wikman, D.Q. Cui, Z.J. Shen, J. Nucl. Mater. 470, 1708 (2016)

[7] L. Liu, Q. Ding, Y. Zhong, J. Zou, J. Wu, Y.L. Chiu, J. Li, Z. Zhang, Q. Yu, Z. Shen, Mater. Today (2018). https://doi.org/10. 1016/j.mattod.2017.11.004 
[8] S. Lenders, M. Thone, A. Riemer, T. Niendorf, T. Troster, H.A. Richard, H.J. Maier, Int. J. Fatigue 48, 300 (2013)

[9] L.C. Zhang, H. Attar, Adv. Eng. Mater. 18, 463 (2016)

[10] K.N. Amato, S.M. Gaytan, L.E. Murr, E. Martinez, P.W. Shindo, J. Hernandez, S. Collins, F. Medina, Acta Mater. 60, 2229 (2012)

[11] D. Wang, C. Yu, X. Zhou, J. Ma, W. Liu, Z. Shen, Appl. Sci. 7, $430(2017)$

[12] Y. Liu, Y.Q. Yang, D. Wang, Int. J. Adv. Manuf. Technol. 87, 647 (2016)

[13] B. Qian, K. Saeidi, L. Kvetkova, F. Lofaj, C. Xiao, Z. Shen, Dent. Mater. 31, 1435 (2015)

[14] X.P. Li, C.W. Kang, H. Huang, L.C. Zhang, T.B. Sercombe, Mater. Sci. Eng. A 606, 370 (2014)

[15] N.J. Harrison, I. Todd, K. Mumtaz, Acta Mater. 94, 59 (2015)

[16] E. Chauvet, P. Kontis, E.A. Jagle, B. Gault, D. Raabe, C. Tassin, J.J. Blandin, R. Dendievel, B. Vayre, S. Abed, G. Martin, Acta Mater. 142, 82 (2018)

[17] A. Huber, A. Arakcheev, G. Sergienko, I. Steudel, M. Wirtz, A.V. Burdakov, J.W. Coenen, A. Kreter, J. Linke, P. Mertens, V. Philipps, G. Pintsuk, M. Reinhart, U. Samm, A. Shoshin, B. Schweer, B. Unterberg, M. Zlobinski, Phys. Scr. T159, 014005 (2014)

[18] N. Farid, D. Zhao, H.Y. Oderji, H. Ding, J. Nucl. Mater. 463, $241(2015)$

[19] M. Wirtz, G. Cempura, J. Linke, G. Pintsuk, I. Uytdenhouwen, Fusion Eng. Des. 88, 1768 (2013)

[20] M. Wirtz, J. Linke, T. Loewenhoff, G. Pintsuk, I. Uytdenhouwen, Phys. Scr. T167, 014015 (2016)

[21] L.N. Carter, C. Martin, P.J. Withers, M.M. Attallah, J. Alloys Compd. 615, 338 (2014)

[22] D.Z. Wang, C.F. Yu, J. Ma, W. Liu, Z.J. Shen, Mater. Des. 129, 44 (2017)
[23] P. Gumbsch, J. Riedle, A. Hartmaier, H.F. Fischmeister, Science 282, 1293 (1998)

[24] L. Qi, D.C. Chrzan, Phys. Rev. Lett. 112, 11 (2014)

[25] L. Romaner, C. Ambrosch-Draxl, R. Pippan, Phys. Rev. Lett. 104, 195503 (2010)

[26] J.J. Xu, X. Lin, P.F. Guo, Y.L. Hu, X.L. Wen, L. Xue, J.R. Liu, W.D. Huang, Mater. Sci. Eng. A 691, 71 (2017)

[27] Y.J. Liu, S.J. Li, H.L. Wang, W.T. Hou, Y.L. Hao, R. Yang, T.B. Sercombe, L.C. Zhang, Acta Mater. 113, 56 (2016)

[28] T. Hirai, G. Pintsuk, J. Linke, M. Batilliot, J. Nucl. Mater. 390-391, 751 (2009)

[29] C.F. Yu, X. Zhou, D.Z. Wang, N. Van Linh, W. Liu, Plasma Sci. Technol. 20, 014019 (2018)

[30] X. Zhou, D.Z. Wang, X.H. Liu, D.D. Zhang, S.L. Qu, J. Ma, G. London, Z.J. Shen, W. Liu, Acta Mater. 98, 1 (2015)

[31] I.M. Mikhailovskij, T.I. Mazilova, V.N. Voyevodin, A.A. Mazilov, Phys. Rev. B 83, 134115 (2011)

[32] C. Mercer, W.O. Soboyejo, Acta Mater. 45, 961 (1997)

[33] X. Zhou, K.L. Li, D.D. Zhang, X.H. Liu, J. Ma, W. Liu, Z.J. Shen, J. Alloys Compd. 631, 153 (2015)

[34] A.M. Chelladurai, K.A. Gopal, S. Murugan, S. Venugopal, T. Jayakumar, Mater. Manuf. Process. 30, 162 (2015)

[35] Y.J. Liu, Z. Liu, Y. Jiang, G.W. Wang, Y. Yang, L.C. Zhang, J. Alloys Compd. 735, 1414 (2018)

[36] P. Mercelis, J.P. Kruth, Rapid Prototyping J. 12, 254 (2006)

[37] Y.L. Li, K. Zhou, P.F. Tan, S.B. Tor, C.K. Chua, K.F. Leong, Int. J. Mech. Sci. 136, 24 (2018)

[38] A.S. Arakcheev, A. Huber, M. Wirtz, G. Sergienko, I. Steudel, A.V. Burdakov, J.W. Coenen, A. Kreter, J. Linke, P. Mertens, A.A. Shoshin, B. Unterberg, A.A. Vasilyev, J. Nucl. Mater. 463, 246 (2015)

[39] K. Kempen, B. Vrancken, S. Buls, L. Thijs, J. Van Humbeeck, J.P. Kruth, J. Manuf. Sci. Eng. 136, 061026 (2014) 